\title{
Tools of Internal Communication from Knowledge Transfer Perspective
}

\author{
- Hudcová Śárka
}

\begin{abstract}
The world nowadays is very often titled as the world of knowledge. Knowledge is a critical differentiator for companies and source of competitive advantage. Therefore companies pay careful attention to knowledge management and put a lot of energy and money to right set-ups ensuring that knowledge is owned and used as adequately as possible. Part of that is also the transfer of knowledge between individuals. The paper deals with the topic of knowledge transfer and sharing and aims to identify the most efficient tool of internal communication in terms of knowledge transfer. The research based on expert interviews is presented in the paper. The results should help management of companies to organize internal communication in the way which allows them to accomplish their knowledge management strategy.
\end{abstract}

Keywords: internal communication, tools of communication, knowledge, knowledge transfer, knowledge sharing, face-to-face communication

JEL Classification: $M 12$

\section{INTRODUCTION}

Nowadays, data and information are everywhere and their amount increases rapidly every year and many companies are trying to build intelligent information systems for analysis and interpretation of the huge amount of data and information. In these times, knowledge and transfer of knowledge are key differentiators in terms of business competitiveness and all successful companies admit that their main objective is to focus on people, their knowledge and they strive to create, improve, transfer/ share, embed, renew the knowledge. In other words, they focus on principles of knowledge management.

Knowledge sharing is particularly important because it is a necessary prerequisite for knowledge application. Nesheim and Gressgard (2014) tested in their research the hypothesis of significant relationship between knowledge sharing and knowledge application and they confirmed the positive relationship. Knowledge sharing supports knowledge application which means "use of knowledge to improve the quality of the work."

Therefore it is becoming critical to understand how to facilitate and improve the process of knowledge sharing and knowledge transfer in order to equip all workers with the right knowledge and to make them ready for usage and application of the knowledge. Knowledge transfer can efficiently work only in the case when right tools of communication are used and when they are used in the right environment and context.

The paper describes the partial result of the bigger research on the role of communication in 
knowledge management. The main objective of the entire research is to design a set of communication tools that supports knowledge management and to define how to use the set in the right way. The first part of the research validated the list of commonly used tools of internal communication in complex, international companies from the IT industry that are recognized as leaders. And the next step (described in the paper) is about assessing the tools in terms of their efficiency towards knowledge transfer. Afterwards, the set will be designed and tested in a selected company.

\section{THEORETICAL BACKGROUND}

Definition of knowledge management by Mladkova (2004) states: „Knowledge management is a conscious activity which results in situation when the right knowledge is possessed by right people at the right time.“ Probst, Raub, and Romhardt (2000) add: „It is vital that knowledge should be shared and distributed within an organization, so that isolated information or experience can be used by the whole company. The key question is: Who needs to know (or be able to do) how much of what, and how can we facilitate knowledge sharing?" And part of the last question creates also the main objective of the paper - what tool of internal communication should be used in order to ensure that knowledge is transferred properly and efficiently.

Every communication represents a knowledge exchange. You cannot avoid this exchange, but you can limit or improve the opportunities for mutual learning. Attempts at secrecy will typically result in mistrust, misjudgements and false information. The best strategy is to share knowledge with the goal of maximizing mutual learning. Naturally, this is easier said than done. (Grof \& Jones, 2003)

Many authors differentiate between knowledge transfer and knowledge sharing. Some consider knowledge sharing to be more because it ensures that knowledge is not only distributed but also understood. Some authors, e.g. Trautman (2014) define knowledge transfer in broader way: „Knowledge transfer means the planned movement of the right skills and information at the right time to keep a workforce productive, competitive, and able to execute business strategy."

The third type of view is simple: "Knowledge transfer and knowledge sharing are sometimes used synonymously or have overlapping content." (Paulin \& Suneson, 2012) For purposes of the paper, knowledge transfer and knowledge sharing are equal terms.

The key question is how to achieve the objective for knowledge transfer in the most efficient way and what kind of tools to use for it. Every author suggests a different criterion and it is impossible to list all of them. Examples are as follows.

- Knowledge is most effectively transferred through interaction. The further one moves from learning through interaction the less likely it is that knowledge will transfer effectively. (Gamble \& Blackwell, 2001)

- Storytelling is highly efficient form of knowledge sharing because it communicates data, contextual rules and subtleties of behaviour that may be difficult to state explicitly. (Bergeron, 2003) 
- What is required for knowledge sharing? Trust, trust and trust! The ability to communicate clearly and with enough bandwidth to transfer meaning. A common context or language. A reason or goal for sharing. The space to think and reflect. The ability to interact with others in a non-purposeful way. The autonomy to share. Awareness that knowledge is local and sticky and often does not transfer easily. A flexible organizational structure that supports knowledge sharing. The infrastructure to support knowledge and information sharing. (Coleman, 1999)

- Knowledge sharing requires two primary and closely interrelated elements, both on which are developed through a process of social interaction and communication. First, and adequate level of trust to be developed between the individuals, ideally with the strongest forms of personal trust being developed. Secondly, people require to develop a basic understanding of the values, assumptions, and viewpoints which underpin each other's knowledge base. (Hislop, 2005)

Probst, Raub, and Romhardt (2000) summarize: "Natural situations for sharing knowledge are those in which colleagues are physically present at the place of work. Where there are fewer opportunities for working together or meeting informally, efforts must be made to arrange social situations in which knowledge can be shared."

Efficient knowledge transfer between employees can be defined as the situation when knowledge is moved from a worker to other one/s and the other worker not only understands the information but is also ready to use the knowledge.

If the knowledge transfer is efficient, it creates basis for knowledge application which aims at improving work quality. Nesheim and Gressgard (2014) explain: "It may involve more timely responses, better quality decisions and improved coordination between activities and team members. In order to apply knowledge across a distributed organization, one is often dependent on sharing and dissemination of the appropriate information. A high level of knowledge sharing behaviour will tend to increase the number of ideas and knowledge elements being discussed."

Patriotta, Castellano, and Wright (2013) bring other and similar perspective: “The high-level managers recognized the benefits of knowledge transfer as a way to avoid duplication of efforts, reduce time and energy in accessing information and ready-made solutions, learn from others' best ways of doing things, and achieve global scale efficiency."

There were some researches identifying the most efficient or important channel of knowledge transfer between universities and industry (Bekkers \& Bodas Freitas, 2008), between countries (Marcon, 2012 or Schleimer \& Riege, 2009), between projects (Frank \& Ribeiro, 2014) or within strategic alliances (Walter \& Lechner \& Kellermanns, 2007). Their findings usually stay on higher level, e.g. in Bekkers, Bodas Freitas (2008) "personal (informal) contacts". It is obvious that good relationship between communicating persons are necessary but there is no detailed explanation how the contacts happen. More ways are possible, e.g. face-to-face meetings, phone conversations, emails, and it is possible to presume that the combination of more tools is actually used.

The objective of the paper is to go deeper and focus on particular tools of internal communication with the intention to identify how efficient the commonly used tools of internal communi- 
cation are for purpose of knowledge transfer. Therefore it is necessary to understand characteristics of the tools. Hislop (2005) brings a summary in the table below.

Tab. 1 - Characteristics of Various Communication Mediums. Source: Hislop (2005)

\begin{tabular}{|c|c|}
\hline Medium & Communication characteristics \\
\hline $\begin{array}{l}\text { Face-to-face } \\
\text { communication }\end{array}$ & $\begin{array}{l}\text { - Information rich (social cues such as facial expressions, voice, gesture } \\
\text { visible). Plus, synchronous communication, potential rapid high-quality } \\
\text { feedback/ interaction } \\
\text { - Most relevant for sharing of tacit knowledge } \\
\text { - Spontaneous/ informal interactions possible when people geographi- } \\
\text { cally proximate } \\
\text { - Conditions amenable to development of trust (other factors excluded) } \\
\text { - Expensive when people geographically dispersed }\end{array}$ \\
\hline $\begin{array}{l}\text { Video } \\
\text { conferencing }\end{array}$ & $\begin{array}{l}\text { - Information rich (social cues, and virtually real time, synchronous me- } \\
\text { dium) } \\
\text { - Expensive to set up } \\
\text { - Set up time inhibits spontaneity }\end{array}$ \\
\hline Telephone & $\begin{array}{l}\text { - Intermediate information richness (tone of voice conveys some social } \\
\text { cues, but gesture, expression invisible. Also synchronous, facilitating } \\
\text { detailed, immediate feedback) } \\
\text { - Cost variable } \\
\text { - Spontaneous/ informal interactions possible irrespective of geographic } \\
\text { proximity } \\
\text { - Can facilitate development of trust where face-to-face interaction dif- } \\
\text { ficult }\end{array}$ \\
\hline e-mail & $\begin{array}{l}\text { - Suitable for sharing of highly codified knowledge } \\
\text { - Relatively low information richness (all social cues lost) } \\
\text { - Inexpensive (cost unrelated to geographic proximity) } \\
\text { - Asynchronous, with variable feedback speed } \\
\text { - Spontaneous/ informal interactions possible irrespective of geographic } \\
\text { proximity } \\
\text { - Permanent record of interactions exists } \\
\text { - Development of trust based on e-mail alone difficult }\end{array}$ \\
\hline
\end{tabular}

The main requirement for efficient knowledge transfer is trust. Trust between communicating people encourages their willingness to share. Sharing itself can be done only through interaction when immediate feedback is ensured. And every communication can be seen as knowledge transfer; however it works much better when it uses specific techniques, e.g. storytelling. 
The best tool for knowledge transfer is face-to-face communication which was pointed out by many authors. However, they admit that needs of the modern world change and face-to-face interaction is not always possible, especially in globally acting companies, so it is worth exploring other tools of internal communication and their characteristics as well. Other question is also about how to properly use the face-to-face communication as it can happen in many different ways and in various environments.

\section{OBJECTIVES AND METHODOLOGY}

The objective of the paper is to assess the most commonly used tools of internal communication according to criteria for efficient knowledge transfer which will result in identification of the most efficient tool of internal communication for purposes of knowledge transfer. Further, it will be defined how the tool should be used in order to achieve the best results for knowledge transfer. The outcome can be applied by organizations in order to support knowledge transfer and thus to improve knowledge management.

The paper presents partial results of the research done for dissertation thesis "The role of communication in knowledge management". The research explores internal communication and knowledge management in selected international IT companies. The part of the research presented in the paper is of qualitative character and is based on expert interviews with four subject matter experts specialized in personnel management and internal communication.

The experts were identified outside the explored companies and all of them meet following criteria: they are educated in the area of personnel management and internal communication (university studies and/or additional courses), they are experienced in the area of personnel management and internal communication (they proved at least 5 years of experience), they know the environment of international IT companies (they are engaged into projects by the companies on regular basis). All experts work for companies providing counselling and training in management, personnel management and some aspects of psychology.

The experts were interviewed individually and in three steps:

1. to identify criteria defining efficiency of knowledge transfer;

2. to assess the most commonly used tools of internal communication in the criteria identified;

3. to analyze the most efficient tool of internal communication for purposes of knowledge transfer in order to define recommendations on how to use the tool.

The qualitative character of the research and the technique of semi-structured interviews were applied in accordance with Bryman and Bell (2003) because the main research questions at this step are about deeper understanding of: how are tools of internal communication in terms of knowledge transfer and how to use face-to-face communication to leverage knowledge transfer.

The theoretical background and a part of the discussion is based on the analysis of references which included three types of references: scientific and expert monographs and collections available in the university library under the key word knowledge management; reviewed scientific and expert articles accessed from the EBSCO service using key words knowledge transfer, knowl- 
edge sharing, knowledge sharing tools, knowledge management and internal communication (Scopus database was prioritized); and finally two articles publically available in web.

The other methods applied in the paper are logical methods of deduction (using theoretical background), induction (drawing conclusions from interviews), analysis (deeper exploration of characteristics of the face-to-face communication via interviews with the experts) and synthesis (building the final outcome from multiple pieces of information/ partial results).

Limitation of the research derives from two major factors: the research is based on answers from four experts and their point of view may not encompass the entire breath and depth of the problem; and the assessment is related to the environment of international companies specialized in IT industry.

\section{RESULTS}

\subsection{Criteria for Efficient Knowledge Transfer}

The first three criteria for efficient knowledge transfer were identified via references analysis. They are: building trust, interaction and story telling application. The other three criteria were identified in interviews with experts: involvement of more senses, spontaneity and balanced dialogue.

- Building trust: a message should be customized for different type of audience; establishing relationships based on mutual trust is key, because "Entry to business relationships, sharing contacts, experience etc. get easier and cheaper with more trust."

- Immediate feedback (interaction): quick and authentic reaction on the communicated message allows flexibility of the communication.

- Involvement of more senses (especially hearing and vision): when a message is communicated using more senses, it gets then stronger, more impressive and more informative.

- Spontaneity in communication usually brings subconscious and uncontrolled messages which enrich the communication with more content.

- Balanced dialogue: both communicating parties should be involved in balanced way, should have time to "tell" and to "listen". No monologue.

- Humour, stories and personal context make a message more interesting and give more impact into it because involves also emotions.

\subsection{Assessment of Tools Used for Internal Communication}

Subject matter experts were asked to assess the most frequently used tools of internal communication according to the criteria above. The scale was $0=$ the worst, $3=$ the best.

The list of tools of internal communication mentioned by Hislop was enlarged with instant messaging, intranet, written communication and web conferences replaced video conferences. These tools were identified in previous research conducted by the author of the paper as the most commonly used tools of internal communication in the Czech Republic. 
Tab. 2 - Assessment of Communication Tools in Key Requirements. Source: own research

\begin{tabular}{|l|c|c|c|c|c|c|}
\hline & $\begin{array}{c}\text { building } \\
\text { trust }\end{array}$ & $\begin{array}{c}\text { immediate } \\
\text { feedback }\end{array}$ & $\begin{array}{c}\text { involvement of } \\
\text { more senses }\end{array}$ & $\begin{array}{c}\text { sponta- } \\
\text { neity }\end{array}$ & $\begin{array}{c}\text { balanced } \\
\text { dialogue }\end{array}$ & $\begin{array}{c}\text { humour, } \\
\text { stories }\end{array}$ \\
\hline email & 1 & 0 & 0 & 1 & 1 & 2 \\
\hline instant messaging & 2 & 2 & 0 & 2 & 1 & 2 \\
\hline intranet & 0 & 0 & 0 & 0 & 0 & 1 \\
\hline $\begin{array}{l}\text { written commu- } \\
\text { nication }\end{array}$ & 0 & 0 & 0 & 0 & 0 & 1 \\
\hline phone calls & 2 & 2 & 0 & 2 & 2 & 2 \\
\hline $\begin{array}{l}\text { face-to-face } \\
\text { communication }\end{array}$ & 3 & 3 & 3 & 3 & 2 & 3 \\
\hline web conferences & 1 & 1 & 2 & 1 & 0 & 1 \\
\hline
\end{tabular}

Records from the table were transferred to a graph. Intranet and written communication were skipped because their values are zero almost in all characteristics.

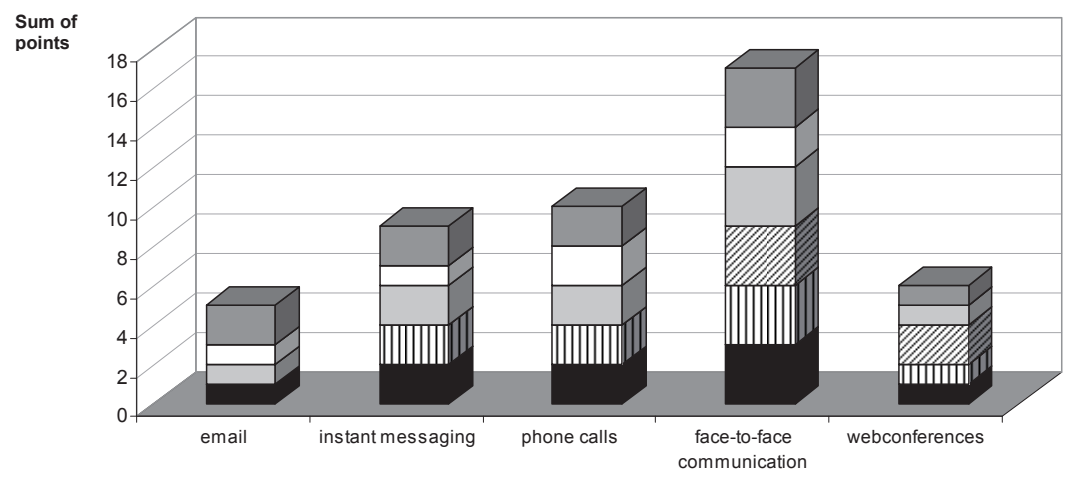

- building trust $\mathbf{m}$ immediate feedback $\square$ involvement of more senses $\square$ spontaneity $\square$ balanced dialogue $\square$ humor, stories

Fig. 1 - Assessment of Communication Tools in Key Requirements. Source: own research

It is obvious that the most efficient tool for knowledge communication is face-to-face communication which means that interviews with experts confirmed statements quoted in references. The more interesting part is to see the sequence of other tools. The second most efficient tools are phone calls and instant messaging; the score for emails and web conferences is the same and the lowest one. (Actually, the lowest score belongs to intranet and written communication that are not presented in the graph).

The interviewed experts added also some comments resulting from their experience and emphasizing the importance of the face-to-face communication:

- "A lot of things can be solved via email, chat, video-conference etc., but when you are solving a problem and sit down together, the solution is found easily and fast. For sure, faster than when you are exchanging ten emails." 
- "If you don't communicate well with clients (both external and internal), you can not build a relationship. In order to build a strong relationship for communication, you have to listen actively. Important is face-to-face communication: you achieve much more when you are a personality, not an email address or a name on a business card."

The face-to-face communication was identified in the selected criteria based assessment as the most efficient tool of internal communication tools for purposes of knowledge transfer and that is why it is worth further exploration.

\subsection{Non-Verbal Communication}

Elements of non-verbal communication enrich face-to-face communication a lot and the question is which elements of non-verbal communication are the most important ones. The four experts were asked to sort types of non-verbal communication according to their contribution to knowledge transfer. They were asked to consider: gestures, facial expressions, haptics, posturology, kinesics, proxemics, eye contact, paralinguistic phenomena, and actions.

They didn't accomplish this task because all of them agreed that it is impossible to work with the types separately, without interaction with others. They said that all the types of non-verbal communication needed to work together, interact and only then, they bring the real value. And this is also the reason why the face-to-face communication is rated so high because it enables almost unlimited transfer of all elements and types of non-verbal communication.

Experts emphasized the communication via actions. Actions and the way how they are done are very often a subject for observation and simulation. The simulation can be conscious or unconscious and is usually focused on model behaviour or actions. This means especially the kind of behaviour/ actions that were recognised by managers or that led to successful achievement of stated goals and objectives.

Unconscious simulation starts spontaneously: employees can see other colleagues who are perhaps more experienced, observe how they handle issues and challenges and start to imitate these actions and behaviour. Sometimes, it is done consciously because employees recognize successful patterns of actions and behaviour. Here, it is very important to make sure that employees simulate and repeat only the good and ethical examples. That is why managers should recognize and award only recommended styles of behaviour and make sure that employees know what is good and what not. It is important to focus not only on the fact whether goals are accomplished but also on the way how they are accomplished.

Conscious simulation is often subject of education activities when people try to simulate desired patterns of acting and behaviour, train handling problems, situations and repeat best practices under supervision by an experienced facilitator or trainer.

Communication via acting is not exclusively related to face-to-face communication. It can be present also in other types of communication. The advantage of the face-to-face communication is that it is possible to observe not only what is done but also how it is done in natural environment.

Experts pointed out to the fact that face-to-face communication is extraordinary also from the spontaneity point of view. Meeting colleagues in an office is very natural and unavoidable. Thus, 
communication between colleagues meeting in the office is more fluent and frequent than communication between colleagues who are distanced and don't work in the same office. Let's consider an example of phone call: it is needed to pick up an earphone, dial a number, wait for a response and it is not sure what the other person is doing, how busy s/he is etc. Then, it opens questions whether the phone call is really needed and it can become more planned and less spontaneous.

Video-conferences can be very similar example. It is possible to transfer a lot of elements of nonverbal communication via video-conferences but on the other hand side, presence of a camera or a recording system eliminates nature and spontaneity in behaviour of participating people. Participants may focus on the camera rather than on what and how they are doing/ saying/ etc.

\subsection{Types of the Face-to-Face Communication}

Face-to-face communication can be classified by using various criteria. For purposes of this paper, it was decided to use following two criteria:

1. Is a meeting planned? The meeting can be planned and scheduled in advance or can be unplanned and unexpected.

2. How many persons do participate in the communication? 2 persons, small group (up to 10 persons), middle and big group (more than 10 persons).

Experts agreed that planned meetings are more valuable in terms of knowledge transfer than unplanned meetings. The reason is that invitees can and should prepare for the planned meeting, planned meetings have a stated objective, are managed by a leader, systematically structured and that is why also more efficient.

When it comes to the group size, experts picked up the small group as the most useful for knowledge transfer. It is because there are more participants present who can share their experience, best practices, can inspire each other by asking questions/ commenting things and thus stimulate communication. The group is still manageable.

The decision between the small group and two-person-meeting was not easy that is why subject matter experts put together a set of criteria, weight for the criteria and did an assessment. The results are presented in the table below.

Tab. 3 - Criteria for Assessment of F2F Communication Types. Source: own research

\begin{tabular}{|l|c|c|c|}
\hline Criterion & Weight & $\begin{array}{c}\text { Assessment: } \\
\text { 2 persons }\end{array}$ & $\begin{array}{c}\text { Assessment: } \\
\text { small group }\end{array}$ \\
\hline Individualized approach to participants & 1 & 2 & 1 \\
\hline Experience sharing, inspiration & 2 & 1 & 2 \\
\hline Impact of communicated content & 1 & 1 & 2 \\
\hline Time spent & 1 & 2 & 1 \\
\hline Total score & & 7 & 8 \\
\hline
\end{tabular}


In the next step, both groups of face-to-face communication were combined and five new types were defined: planned meeting of two persons, planned meeting of a small group, unplanned meeting of two persons, unplanned meeting of a small group, mass communication which is typically planned. All the types were sorted by contribution to knowledge transfer and frequency of occurrence in internal communication.

Frequency of occurrence was defined based on structured observation in selected organizations and based on experience of experts. It presents how particular ways of the face-to-face communication are actually used.

The final order by contribution to knowledge transfer and frequency of occurrence in internal communication is presented in the graph below. The scale from 1 to 5 was used in the figure below ( 1 = the best contribution to knowledge transfer/ the most frequently occurred way, $5=$ the worst contribution to the knowledge transfer/ the least frequently used way).

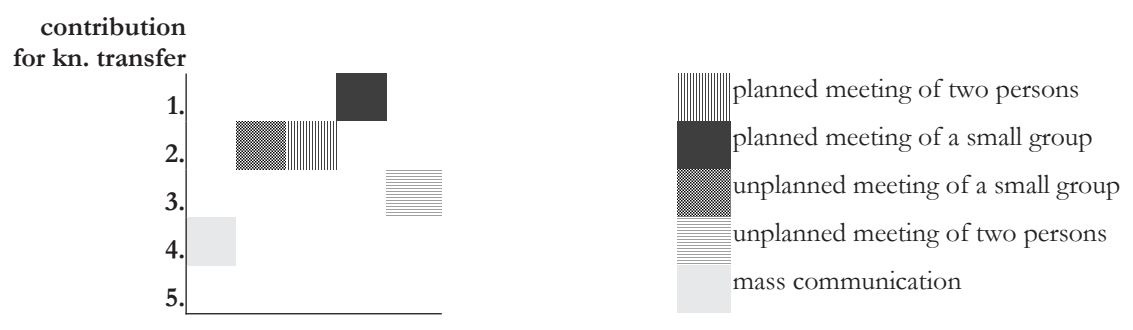

5. 4. 3. 2. 1. frequency of occurrence

Fig. 2 - F2F Communication Types by Contribution to Knowledge Transfer and Frequency of Occurrence. Source: own research

The optimum status is the 1.1 position and it is obvious that there are two types very close to this positions: planned meeting of a small group and unplanned meeting of a small group. In order to bring them to the higher level and to increase their contribution to overall knowledge transfer in an organisation, it is recommended to take following actions:

- To increase the frequency of occurrence of planned meetings of small groups. In other words, to support regular meetings of functional and project teams, communities of practise; to make all teams to meet more often in face-to-face environment, etc.

- To leverage contribution for knowledge transfer in unplanned meetings of two persons which can be achieved through adopting right tools and techniques (mentoring, coaching, observation - simulation, structured dialogue, story telling) into standard acting and behaviour.

For purposes of exploring the face-to-face communication deeply, types of the face-to-face communication where defined based on two criteria: planned in advance and number of participants. These types were assessed by the group of experts in terms of knowledge transfer (planned meetings are more valuable than unplanned and a small group is better than a big group) and frequency of occurrence. The most efficient type of the face-to-face communication from the 
knowledge sharing perspective is planned meetings of small groups. Unplanned meetings of two persons are the most common, so it is highly recommended to leverage knowledge transfer through using right techniques in this context.

\section{DISCUSSION}

There are many researches conducted in the area of knowledge management and knowledge transfer/ sharing within an organization (e.g. Nesheim \& Gressgard, 2014, Frank \& Ribeiro, 2014, Fang, Yang \& Hsu, 2013, Schleimer \& Riege, 2009) as this topic is critical from many perspectives, e.g. competitiveness in the market. Typically, the researches are focused on higher level concepts and don't explore particular tools and communication channels.

All the most commonly used tools of internal communication were assessed by the group of experts in the area of personnel management and internal communication according to defined criteria determining efficiency of knowledge transfer. The face-to-face communication was identified as the most efficient tool in terms of knowledge sharing. This is strongly supported by Probst, Raub, and Romhardt (2000) and some recent researches, e.g. Ryan, O'Connor (2013) who proved direct correlation between face-to-face social interaction and knowledge sharing.

Face-to-face communication was explored more into depth. Unplanned meetings of two persons and planned meetings of small groups were pointed out as particularly important which corresponds with the case study by Patriotta, Castellano, and Wright (2013). Properly planned working meetings are considered strong knowledge sharing tool also by Lopez-Fresno \& Savolainen (2014) and they emphasize the proper planning and preparation part in order to achieve the real value (knowledge sharing).

All the assessment was done with consideration of typical situations and specific circumstances for communication were not taken into account. Same results in knowledge transfer efficiency can be achieved in more ways depending on the particular context. Apart from that, the research described in the paper is limited with other factors: limited number of respondents and focus on international organisations from IT industry. The other thing can be about limiting the research to qualitative character and using semi-structured interviews as the main research technique. This is caused by the fact that only partial results of bigger research are presented in the paper. Some limitations will be eliminated in further steps when the results will be consolidated into a proposed set of communication tools, inclusive defined guidance for their usage, and this will be tested in practice.

For practical application of the results in organisations, it is important to understand that tools of communication are not applied in isolation but are part of the overall concept of internal communication which results from organizational strategy and culture. In order to support the right outcomes, it is necessary to create also the right environment and conditions for the communication aimed at knowledge transfer and sharing. The range of factors is very wide, e.g. processes and rules in the organization, atmosphere, set up of offices and tables, etc. This area was already investigated (e.g. Nesheim \& Gressgard, 2014) but still provides a lot of space for exploration and it is subject of next research steps of the author as well. 


\section{CONCLUSION}

The research confirmed that face-to-face communication is the most efficient tool of internal communication in terms of knowledge transfer. In cases when it is not possible to use the faceto-face communication, other valuable tools of internal communication are: phone calls, instant messaging, web conferences and emails. Tools are sorted by their efficiency in terms of knowledge transfer.

The primary role of the face-to-face communication is given especially by two features: it enables transfer of a lot of messages because of non-verbal elements contained in it; it supports nature and spontaneity in reactions of all present parties. Communication via acting plays key role here as well. Actions are very often observed and imitated which can happen both consciously and unconsciously.

The face-to-face communication can be planned or unplanned and different number of participants can be present. Experts highlight planned meetings of small groups and unplanned meetings of two persons for purposes of knowledge transfer. They recommend enhancing their efficiency by increased frequency of meetings and by the usage of right tools and techniques that should be adopted as natural elements of all employees acting and behaviour.

\section{References}

1. Bergeron, B. (2003). Essentials of Knowledge Management. New Jersey: John Wiley and Sons.

2. Bekkers, R., \& Bodas Freitas, I. M. (2008). Analysing knowledge transfer channels between universities and industry: To what degree do sectors also matter? Research Policy, 37(10), 1837 - 1853. http://dx.doi.org/10.1016/j.respol.2008.07.007

3. Bryman, A., \& Bell, E. (2003). Business research methods. Oxford: Oxford University Press.

4. Coleman, D. (1999). Groupware: Collaboration and Knowledge Sharing. Knowledge Management Handbook. Boca Raton: CRC Press LLC.

5. Fang, S. -C., Yang, C. -W., \& Hsu, W. -Y. (2013). Inter-organizational knowledge transfer: the perspective of knowledge governance. Journal of Knowledge Management, 17(6), 943 - 957. http://dx.doi.org/10.1108/jkm-04-2013-0138

6. Frank, A. G., \& Ribeiro, J. L. D. (2014). An integrative model for knowledge transfer between new product development project teams. Knowledge Management Research and Practice, 12(2), 215 - 225. http://dx.doi.org/10.1057/kmrp.2012.57

7. Gamble, P., \& Blackwell, J. (2001). Knowledge Management. A state of the art guide. London: Kogan Page.

8. Groff T. R., \& Jones T. P. (2003). Introduction to Knowledge Management. Burlington: Butterworth-Heinemann.

9. Hislop, D. (2005). Knowledge Management in Organizations. A Critical Introduction. Oxford: Oxford University Press.

10. Lopez-Fresno, P., \& Savolainen, T. (2014). Working meetings - a Tool for Building or Destroying Trust in knowledge Creation and Sharing. Electronic Journal of Knowledge Management, 12(2), 130 - 136. 
11. Marcon, M. R. (2012). SMEs and the internationalization of R\&D activities: Knowledge transfer flows between firms. European Journal of International Management, 6(2), 133 - 153. http://dx.doi.org/10.1504/ejim.2012.045794

12. Mladkova, L. (2004). Management ₹nalostí v praxi. Praha: Professional Publishing.

13. Nesheim, T., \& Gressgard, L. J. (2014). Knowledge sharing in a complex organization: Antecedents and safety effects. Safety Science, 62, 28 - 36. http://dx.doi.org/10.1016/ j.ssci.2013.07.018

14. Patriotta, G., Castellano, A., \& Wright, M. (2013). Coordinating knowledge transfer: Higher managers as higher-level intermediaries. Journal of World Business, 48(4), 515 - 526. http:// dx.doi.org/10.1016/j.jwb.2012.09.007

15. Paulin, D., \& Suneson, K. (2012). Knowledge Transfer, Knowledge Sharing and Knowledge Barriers. EJKM 10(1), 321. Retrieved from www.ejkm.com/issue/download.html?idArticle=321.

16. Probst, G., Raub, S., \& Romhardt, K. (2000). Managing Knowledge. Building Blocks for Success. New York: John Wiley and Sons.

17. Ryan, S., \& O'Connor, R. V. (2013). Acquiring and sharing tacit knowledge in software development teams: An empirical study. Information and Software Technology, 55(9), 1614 - 1624. http://dx.doi.org/10.1016/j.infsof.2013.02.013

18. Schleimer, S., \& Riege, A. (2009). Knowledge transfer between globally dispersed units at BMW. Journal of Knowledge Management, 13(1), 27 - 41. http://dx.doi.org/10.1108/1367327091 0931143

19. Trautman, S. (2014). 4 Practices to Embed Knowledge Transfer into Your Business Culture. Retrieved June 19, 2014, from http://stevetrautman.com/4-practices-to-embed-knowledge-transferinto-your-business-culture/.

20. Walter, J., Lechner, Ch., \& Kellermanns, F. W. (2007). Knowledge transfer between and within alliance partners: Private versus collective benefits of social capital. Journal of Business Research, 60(7), 698 - 710. http://dx.doi.org/10.1016/j.jbusres.2007.01.026

\section{Contact information}

Ing. Śárka Hudcová

Faculty of Economics and Management

Czech University of Life Sciences

Kamýcká 129, 16521 Prague, Czech Republic

Email:sarka.budcova@seznam.cr. 